\title{
Which crucial measures do patients need to follow to prevent transmission of COVID-19 in the dental setting?
}

\section{Silvia Spivakovsky ${ }^{1}$}

\section{A Commentary on}

Siles-Garcia A A, Alzamora-Cepeda A G, Atoche-Socola K J,

Peña-Soto C, Arriola-Guillén L E.

Biosafety for dental patients during dentistry care after COVID-19:

a review of the literature. Disaster Med Public Health Prep 2020;

DOI: $10.1017 / \mathrm{dmp} .2020 .252$.

\section{Abstract}

Data sources Medline via PubMed, Scopus, Science Direct, Scielo and Google Scholar were searched without language restriction until 28 May 2020.

Study selection Publications on the topic of biosafety measures before, during and after dental practice from observational studies, systematic reviews and literature reviews were included, while letters to the editor, individual opinions and books were excluded.

Data extraction and synthesis The authors used a narrative review to describe the findings and grouped them into two categories: those considerations before dental care and those during dental consultation.

Results The review was based on 43 publications. Of those, 23 were recent reviews, guidelines, protocols and recommendations from national and international organisations; three were COVID-related original studies and the remainder were pre-COVID publications on handpieces, surface contamination, ventilation, aerosols and airborne spread, ultrasonics, hand washing and dental pain management.

Conclusions Patients should conform to COVID-19 screening protocols in order to receive dental care and follow all the procedures in place to prevent transmission while in the dental office.
Practice point

- Patients need to follow strict protocols before, during and after dental care in order to reduce the risk of transmission of COVID19 that could take place during the dental visit.

\section{Commentary}

With new information published each day on COVID-19, it is at times overwhelming and even confusing to determine what the healthcare professional should and can do and implement to continue providing safe treatment to patients.

The review attempts to summarise the current knowledge behind the strategies that should be implemented to minimise the risk of transmission. which starts before the patient comes to the office but also during and after.

The authors searched multiple databases, without language restrictions, and aimed to include literature reviews and systematic reviews. Twenty-three of the references used in the review came from reviews, recommendations and protocols specific to COVID-19 from national and international organisations, mainly published in English but also some in Spanish.

By virtue of being a review of reviews, it not clear where some of the statements originated from, but also it is possible that some of the references may be missing. As a result, there is the potential for creating frustration among readers looking for the original data, an example being their review on the use of rinses.

The authors stated the need to develop organised protocols that include detail information for patients to follow, although that information is part of multiple publications cited.

Because of the uncertainty regarding the number of asymptomatic carriers and the role of immunity related to COVID19 , it is crucial to implement procedures to minimise the risk of transmission. Dentists must comply with local and national guidelines and protocols, and can rely on publications from local and national dental organisations to keep up to date.

\section{Author affiliation \\ ${ }^{1} N Y U$ College of Dentistry, NY, US}

Evidence-Based Dentistry (2020) 21, 79. https://doi.org/ 10.1038/s41432-020-0116-4 